\title{
Prevalence of meconium ileus marks the severity of mutations of the Cystic Fibrosis Transmembrane Conductance Regulator (CFTR) gene
}

\author{
Annie Dupuis, $\mathrm{PhD}^{1,2}$, Katherine Keenan, $\mathrm{BSc}^{3}$, Chee Y. Ooi, MBBS, PhD ${ }^{4,5}$, Ruslan Dorfman, $\mathrm{PhD}^{6}$, \\ Marci K. Sontag, PhD ${ }^{7}$, Lutz Naehrlich, $\mathrm{MD}^{8}$, Carlo Castellani, MD ${ }^{9}$, Lisa J. Strug, PhD, ScM ${ }^{2}, 10$, \\ Johanna M. Rommens, PhD ${ }^{10,11}$ and Tanja Gonska, MD ${ }^{2,12}$
}

Rationale: Meconium ileus (MI) is a perinatal complication in cystic fibrosis $(\mathrm{CF})$, which is only minimally influenced by environmental factors. We derived and examined MI prevalence (MIP) scores to assess CFTR phenotype-phenotype correlation for severe mutations.

Method: MIP scores were established using a Canadian CF population $(n=2,492)$ as estimates of the proportion of patients with MI among all patients carrying the same CFTR mutation, focusing on patients with p.F508del as the second allele. Comparisons were made to the registries from the US CF Foundation $(n=43,432)$, Italy (Veneto/Trentino/Alto Adige regions) $(n=1,788)$, and Germany $(n=3,596)$.

Results: The prevalence of MI varied among the different registries (13-21\%). MI was predominantly prevalent in patients with pancreatic insufficiency carrying "severe" CFTR mutations. In this severe spectrum MIP scores further distinguished between mutation types, for example, G542X (0.31) with a high, F508del (0.22) with a moderate, and G551D (0.08) with a low MIP score. Higher MIP scores were associated with more severe clinical phenotypes, such as a lower forced expiratory volume in 1 second $(P=0.01)$ and body mass index $z$ score $(P=0.04)$.

Conclusions: MIP scores can be used to rank CFTR mutations according to their clinical severity and provide a means to expand delineation of CF phenotypes.

Genet Med advance online publication 18 June 2015

Key Words: cystic fibrosis; cystic fibrosis transmembrane conductance regulator gene; genotype-phenotype study; meconium ileus prevalence; pancreas insufficiency prevalence

\section{INTRODUCTION}

Meconium ileus (MI) is the earliest disease complication in cystic fibrosis (CF), a multiorgan disease that is caused by mutations in the cystic fibrosis transmembrane conductance regulator (CFTR) gene. ${ }^{1}$ Affected newborns often present with severe bowel obstruction caused by inspissated mucous and meconium occluding the mid or distal small bowel. Resolution of the ileal obstruction requires extensive treatment, including rectal infusion of gastrografin and/or enema under fluoroscopic control, as well as additional saline/acetylcysteine enemas. In about half of the newborns with CF presenting with MI, surgical intervention is necessary to alleviate the blockage and/or manage secondary complications such as intestinal atresia or intestinal perforation. Thanks to advancements in surgical and medical management, the outcome of infants with $\mathrm{CF}$ and MI has greatly improved, and their survival is now similar to children with $\mathrm{CF}$ without MI.,3

Only a subset of patients with CF develop MI, and previous studies have reported an MI prevalence (MIP) of $14 \%$ in
Canada $^{4} 14 \%$ in Italy ${ }^{5}$ and $20 \%$ in the United States. ${ }^{6}$ Further, MI demonstrates notable heritability. ${ }^{7,8}$ Although studies have shown that non-CFTR genes contribute to susceptibility, the CFTR genotype itself affects the occurrence of this complication; only patients with the more severe CFTR variants are at risk for MI.

More than 1,900 CFTR variants have been identified (http://www.genet.sickkids.on.ca) in patients with CF. Attempts to predict the functional severity of these mutations or their predictive value on the overall clinical outcome of individual patients have been challenged by a general lack of genotype and phenotype correlation. ${ }^{4,10,11}$ Lung disease, the primary determinant of a patient's CF disease course, shows great heterogeneity, even among patients carrying the same CFTR mutations, because of the impact of environmental factors and multiple modifier genes. ${ }^{4}$ Exocrine pancreatic disease, however, correlates well with the underlying genotype, ${ }^{12,13}$ and $\mathrm{CF}$ has traditionally been dichotomized

${ }^{1}$ Clinical Research Services, Research Institute, The Hospital for Sick Children, Toronto, Ontario, Canada; ${ }^{2}$ Dalla Lana School of Public Health, University of Toronto, Toronto, Ontario, Canada; ${ }^{3}$ Program in Physiology and Experimental Medicine, Research Institute, The Hospital for Sick Children, Toronto, Ontario, Canada; ${ }^{4}$ Discipline of Paediatrics, School of Women's and Children's Health, Faculty of Medicine, University of New South Wales, Sydney, Australia; ${ }^{5}$ Department of Gastroenterology, Sydney Children's Hospital Randwick, New South Wales, Australia; ${ }^{6}$ GeneYouIn Inc., Toronto, Ontario, Canada; ${ }^{7}$ Department of Epidemiology, Colorado School of Public Health, University of Colorado Denver, Anschutz Medical Campus, Aurora, Colorado, USA; ${ }^{8}$ Department of Pediatrics, Justus-Liebig-University Giessen, Giessen, Germany; ${ }^{9} \mathrm{Cystic}$ Fibrosis Center, Azienda Ospedaliera Universitaria Integrata, Verona, Italy; ${ }^{10}$ Program in Genetics \& Genome Biology, Research Institute, The Hospital for Sick Children and Division of Biostatistics; ${ }^{11}$ Department of Molecular Genetics, University of Toronto, Toronto, Ontario, Canada; ${ }^{12}$ Department of Pediatrics, University of Toronto, Toronto, Ontario, Canada. Correspondence: Tanja Gonska (tanja. gonska@sickkids.ca)

Submitted 20 January 2015; accepted 1 May 2015; advance online publication 18 June 2015. doi:10.1038/gim.2015.79 
into a pancreatic insufficient (PI) and thus "severe" CF phenotype or a pancreatic sufficient (PS) and thus "mild" CF phenotype. In general, this distinction corresponds well to the mutation class system, first proposed in 1993 (ref. 14), that considered the underlying molecular consequences for the CFTR protein. According to this system, class I-III mutations, which include most disease alleles, are associated with minimal or no CFTR function and often are associated with a PI phenotype. Class IV and V mutations confer residual CFTR function and are associated with a PS phenotype. ${ }^{12,13,15}$

Recently established PI prevalence (PIP) scores are being used to distinguish more accurately between the severity of different CFTR mutations within the milder range. ${ }^{16,17}$ By contrast, $\mathrm{MI}$ is commonly reported in patients with CF carrying "severe" class I-III CFTR mutations. ${ }^{4}$ Given the early presentation with minimal opportunity for environmental influence, we sought to determine whether MI is associated with the CFTR genotype using large patient cohorts. We hypothesized that, similar to the pancreatic phenotype, ${ }^{16,18}$ the susceptibility to MI is influenced by specific CFTR genotypes, and that the prevalence of MI can be used to discriminate among severe CFTR mutations. We estimated and compared the prevalence of MI for different CFTR mutations using data sets from four countries and investigated association with other clinical markers of disease severity, including lung function (forced expiratory volume in 1 second $\left(\mathrm{FEV}_{1}\right)$ ) and body mass index (BMI).

\section{Study cohorts}

\section{MATERIALS AND METHODS}

The Canadian Gene Modifier Consortium (GMC) was established to carry out large-scale genetic studies with recruitment of $60 \%$ of Canadian patients with CF between 2004 and 2008 $(n=2,492)$ and has been described earlier. ${ }^{19}$ In addition, we obtained longitudinal lung function and nutritional data from a similarly aged patient population from the Canadian patient data registry including only patients born after $1980(n=3,153)$. Further, we worked with the US patient registry $(n=43,432)$, the German CF patient registry $(n=3,596)$ and the CF patient data set from Veneto/Trentino/Alto Adige (northern Italy; $n=$ 1,788 ) including patients with information about MI status.

\section{Data collection}

The following data were collected from all groups: occurrence of MI, age at CF diagnosis, CFTR genotype, and presence of siblings with CF. Additional data were collected from the Canadian patient data registry, including longitudinal lung function measures (percentage of predicted $\mathrm{FEV}_{1}\left(\mathrm{FEV}_{1}\right.$ \%pred)), weight and height for patients born in or after 1980 (reporting years 19862013). Lung function and growth data were obtained from the first stable clinic visit per year. All available data were included in our analysis.

The study was approved by the Research Ethics Board of The Hospital for Sick Children (Toronto, Canada), Cystic Fibrosis Canada, CF Foundation (Bethesda, MD), and the German and Italian CF patient registry committees.

\section{Data validation and establishing scores}

MIP score. The GMC cohort, which provided the most detailed phenotype data, was used to investigate the accuracy of the MI phenotype. We reviewed the charts of a total of 227 patients with CF with a diagnosis of MI across Canadian CF centers in the database and for whom charts were available. Further, we reviewed the charts of all 42 patients from The Hospital for Sick Children who were classified as not having MI and who received a diagnosis of $\mathrm{CF}$ at $\leq 2$ months of age. The MI phenotype was verified based on a physician's note and/or imaging results documenting the MI. It was considered false in the absence of any chart documentation for MI and/or when chart documentation suggested that other disease symptoms had led to a diagnosis of CF. We discovered that the age at CF diagnosis was helpful in confirming the presence or absence of MI. False assignment of MI when a patient with CF in fact did not have MI occurred in $15.8 \%$ of the cases. It was lower when the CF diagnosis was established before 1 (1.65\%) or 2 months of age (11.1\%) and higher when the CF diagnosis was established after 3 months of age (41.2\%). The opposite was true for falsely assigning the non-MI variable to a patient with CF, which occurred in $19 \%$ of cases. It was higher among patients with a CF diagnosis before 1 month of age (32\%) in the absence of any siblings with a CF diagnosis, but lower with a CF diagnosis between the ages of 1 and 2 months (4\%).

In view of these findings, we used logistic regression analysis to model the probability of MI using the validated status obtained from the chart review as the outcome and the reported diagnosis of MI, age at diagnosis, and the presence/ absence of a sibling with CF obtained from the database as predictors. The coefficients from the model then were applied to estimate the probability of MI in the data sets obtained from the GMC as well as the Canadian, US, German, and North Italian patient registries. The probability term is provided in the Supplementary Methods online.

A MIP score was estimated for each CF-causing variant as the average probability of MI for individuals with the CF-causing variant on one allele and F508del on the second allele. MIP scores were calculated for CF-causing variant groups populated by at least 10 patients with CF within each country group. While the absence or presence of siblings with CF was a component of the MI probability term, the siblings themselves were not identifiable to each other, risking confounding the MIP scores for CFTR mutations with a small number of patients. However, we controlled all small groups of patients carrying the same CFTR mutation on the second allele within the GMC cohort and confirmed a similar MI frequency between groups of patients with and without siblings.

PIP score. The GMC cohort was used to develop PIP scores, as previously described. ${ }^{18}$ Exocrine pancreatic function was evaluated using one or more of the following tests, and PI was diagnosed when there was a low serum cationic trypsinogen concentration $(<16.5 \mathrm{ng} / \mathrm{ml})$, low fecal elastase concentration in stool $(<200 \mu \mathrm{g} / \mathrm{g}$ stool), low isoamylase concentration 
( $<13$ IU/l), high 72-h fecal fat $(>7 \%)$ and/or abnormal pancreas imaging at $\geq 10$ years of age.

The PIP score was calculated only for the GMC cohort as the proportion of PI among all patients (PI and non-PI) carrying the same CF-causing variants (where $n \geq 10$ ).

\section{Longitudinal analysis of lung function and BMI}

$\mathrm{FEV}_{1} \%$ pred was determined using the equation presented by Hankinson et al. ${ }^{20}$ and Wang et al. ${ }^{21}$ to adjust for age, sex, and height. BMI $z$ scores (zBMI) were derived from height, weight, age, and sex using the SAS macros obtained from the Centers for Disease Control and Prevention growth percentiles ( http:// www.cdc.gov/nccdphp/dnpao/growthcharts/resources/sas. htm; accessed 26 March 2014). Both Canadian patient databases - the GMC and the Canadian patient data registry-were used to model the effect of MIP score and individual MI status on $\mathrm{FEV}_{1}$ \%pred and $\mathrm{zBMI}$ across ages 6-30 years using hierarchical linear regression. ${ }^{22}$ Models controlled for pancreatic status, sex, and age. We also examined interactions with age to assess the effect of each variable on the rate of change of $\mathrm{FEV}_{1}$ \%pred and zBMI over age. SAS version 9.3 was used for statistical analysis (SAS Institute, Cary, NC).

\section{RESULTS}

\section{MI prevalence}

Given the frequencies of CF-associated variants and the prominence of F508del as the most common CF-associated allele, we focused on patients with CF with heterozygous genotypes including at least one F508del allele. The MI prevalence among patients carrying F508del on one allele ranged from 13\% in the North Italian and $15 \%$ in the Canadian to $20 \%$ in the German and $21 \%$ in the US CF patient registry cohorts; the MIP calculated using the predicted probability of MI led to modestly higher results (Figure 1).

\section{MIP scores in the GMC cohort}

CF-causing variants were assigned individual MIP scores (Table 1). MI occurred only in patients carrying "severe" CFTR mutations, which were associated with a predominantly PI phenotype (high PIP scores). Within this severe end of the spectrum (PIP > 0.25) MIP scores ranged from 0.0 to 0.31 , indicating that individual "severe" CFTR mutations confer different levels of risk for this early occurring CF disease complication. By contrast, the risk for MI, and thus the MIP score, was low or zero for CF-causing variants, associated with a predominantly PS phenotype (PIP < 0.25).

\section{International comparisons of MIP scores}

To evaluate whether CF-causing variants are associated with MIP scores and do not merely reflect population or environmental conditions, MIP scores were estimated for the most frequent CFTR mutations in the US, North Italian, and German CF patient registries (Table 1 ). The MIP scores derived from the GMC cohort correlated very well with the MIP scores estimated

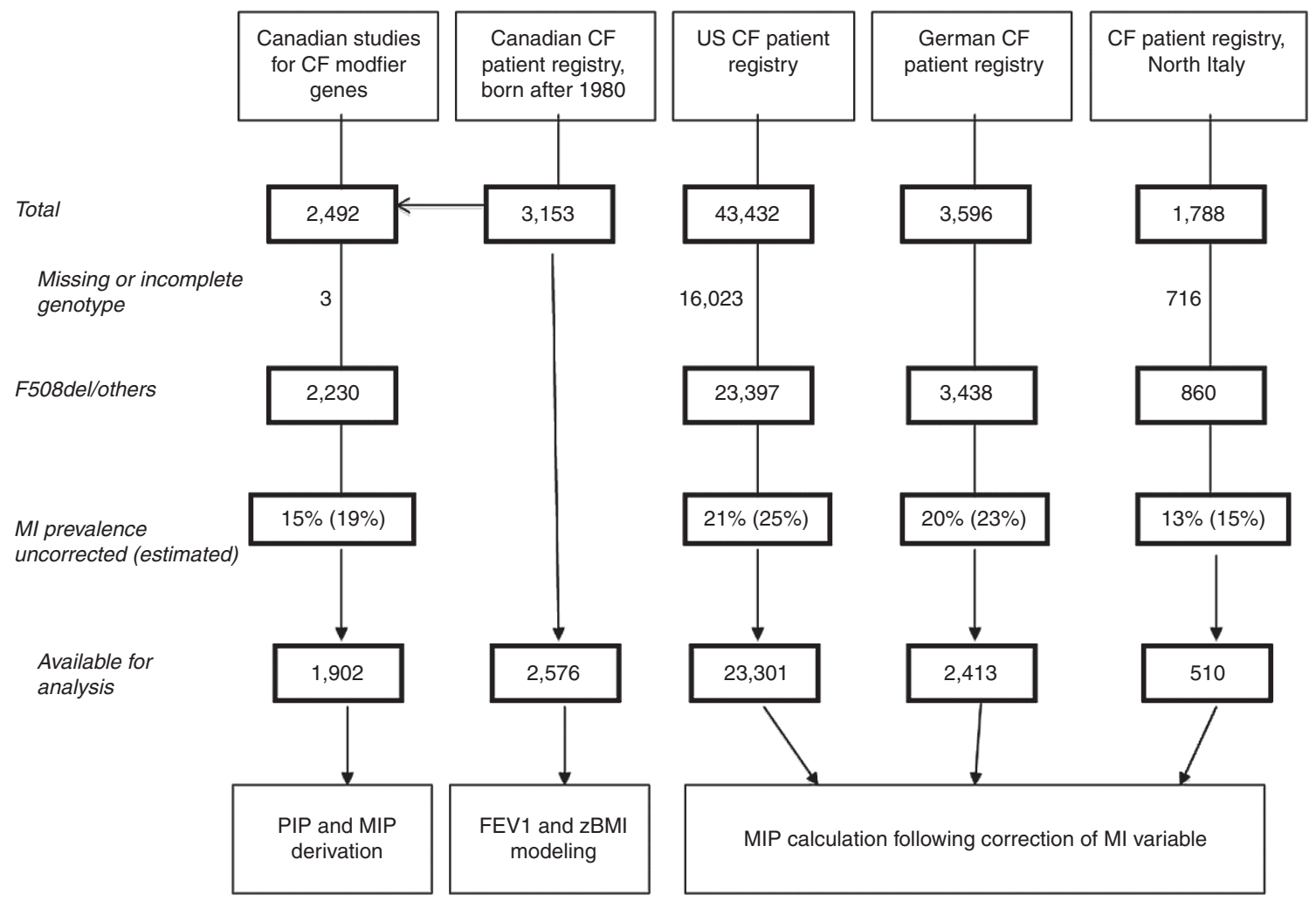

Figure 1 Overview of the different databases and cystic fibrosis (CF) patient data registries. Only patients with F508del and a known second mutation, as well as with known age at CF diagnosis and meconium ileus variable, were included in the analysis. 
Table 1 Meconium ileus prevalence scores for the most common cystic fibrosis-causing variants

\begin{tabular}{|c|c|c|c|c|c|c|c|}
\hline \multicolumn{2}{|c|}{ p. F508del/other variants } & \multirow[b]{2}{*}{ Class } & \multirow{2}{*}{$\begin{array}{c}\text { PIP Canada, } \\
\text { (n) }\end{array}$} & \multicolumn{4}{|c|}{ MIP, (n) } \\
\hline HGVS & Legacy name & & & Canada & United States & Germany & Italy \\
\hline c.262_263delTT & 394delTT & I & & & $0.38(50)$ & & \\
\hline c. $3472 C>T$ & R1158X & I & & & $0.37(35)$ & & \\
\hline c. $1558 \mathrm{G}>\mathrm{T}$ & V520F & & & & $0.35(43)$ & & \\
\hline c. $3484 C>T$ & R1162X & I & & & $0.34(135)$ & $0.17(14)$ & $0.22(45)$ \\
\hline c.2012delT & 2143delT & I & & & $0.33(13)$ & & \\
\hline c. $3276 C>A$ or $G$ & Y1092X & I & $0.92(13)$ & $0.09(12)$ & $0.33(55)$ & & \\
\hline c. $3846 \mathrm{G}>\mathrm{A}$ & W1282X & I & $1.00(13)$ & $0.29(13)$ & $0.32(442)$ & $0.17(20)$ & \\
\hline c. $1477 C>T$ & Q493X & I & $1.00(11)$ & $0.19(11)$ & $0.32(102)$ & & \\
\hline c.3528delC & 3659delC & I & & & $0.31(139)$ & & \\
\hline c. $579+1 \mathrm{G}>\mathrm{T}$ & $711+1 \mathrm{G}>\mathrm{T}$ & & $0.97(39)$ & $0.30(38)$ & $0.31(54)$ & & \\
\hline c. $178 \mathrm{G}>\mathrm{T}$ & E60X & I & & & $0.30(66)$ & & \\
\hline c. $1657 C>T$ & R553X & I & $1.00(16)$ & $0.28(16)$ & $0.30(415)$ & $0.24(107)$ & \\
\hline c. $1585-1 \mathrm{G}>\mathrm{A}$ & $1717-1 \mathrm{G}>\mathrm{A}$ & I & $1.00(12)$ & $0.23(12)$ & $0.29(367)$ & $0.22(38)$ & $0.16(22)$ \\
\hline c. $1766+1 \mathrm{G}>\mathrm{A}$ & $1898+1 \mathrm{G}>\mathrm{A}$ & & & & $0.29(139)$ & & \\
\hline c. $1624 G>T$ & G542X & I & $0.99(73)$ & $0.31(72)$ & $0.29(976)$ & $0.21(79)$ & $0.22(33)$ \\
\hline c.1521_1523delCTT & F508del & ॥ & $0.99(1292)$ & $0.22(1260)$ & $0.27(15391)$ & $0.21(1910)$ & $0.20(230)$ \\
\hline c. $1679 \mathrm{G}>\mathrm{C}$ & R560T & ॥ & & & $0.27(123)$ & & \\
\hline c.3744delA & 3876delA & & & & $0.27(22)$ & & \\
\hline c. $2128 \mathrm{~A}>\mathrm{T}$ & K710X & I & & & $0.26(12)$ & & \\
\hline c.1519_1521delATC & I507del & II & $1.00(20)$ & $0.21(19)$ & $0.25(162)$ & & \\
\hline c. 3909 C >G & N1303K & $\|$ & $0.98(40)$ & $0.13(39)$ & $0.25(534)$ & $0.23(80)$ & $0.14(62)$ \\
\hline c. $489+1 \mathrm{G}>\mathrm{T}$ & $621+1 G>T$ & I & $1.00(90)$ & $0.24(88)$ & $0.25(369)$ & $0.21(11)$ & \\
\hline c. $3266 G>A$ & W1089X & I & & & $0.25(17)$ & & \\
\hline c. $1675 G>A$ & A559T & & & & $0.24(21)$ & & \\
\hline c. $988 \mathrm{G}>\mathrm{T}$ & G330X & & & & $0.24(10)$ & & \\
\hline c.3773_3774insT & 3905insT & & & & $0.23(78)$ & & \\
\hline c. $2988+1 G>A$ & $3120+1 G>A$ & & & & $0.22(121)$ & & \\
\hline c. $443 \mathrm{~T}>\mathrm{C}$ & 1148T;3199del6 & & $1.00(15)$ & $0.22(15)$ & & & \\
\hline c.2052delA & 2184delA & I & & & $0.21(89)$ & $0.22(10)$ & \\
\hline c.2051_2052delAAinsG & $2183 A A>G$ & & & & $0.20(73)$ & & $0.20(42)$ \\
\hline c.948delT & 1078delT & & & & $0.19(20)$ & & \\
\hline c. $1652 \mathrm{G}>\mathrm{A}$ & G551D & III & $0.96(54)$ & $0.08(53)$ & $0.15(979)$ & $0.09(84)$ & \\
\hline c. $254 \mathrm{G}>\mathrm{A}$ & G85E & & $0.50(24)$ & $0.06(24)$ & $0.14(137)$ & & $0.00(10)$ \\
\hline c. $3196 C>T$ & R1066C & & & & $0.14(42)$ & & \\
\hline c. $1466 C>A$ & S489X & & $1.00(14)$ & $0.14(14)$ & & & \\
\hline c. $3808 \mathrm{G}>\mathrm{A}$ & D1270N & & & & $0.13(19)$ & & \\
\hline c. $1055 \mathrm{G}>\mathrm{A}$ & R352Q & & & & $0.12(18)$ & & \\
\hline c. $579+5 G>A$ & $711+5 G>A$ & & & & & & $0.12(30)$ \\
\hline c.2175_2176insA & 2307insA & & & & $0.11(24)$ & & \\
\hline c. $349 C>T$ & R117C & & & & $0.10(37)$ & & \\
\hline c. $1040 \mathrm{G}>\mathrm{C}$ & R347P & IV & $0.18(11)$ & $0.19(11)$ & $0.10(130)$ & $0.02(56)$ & \\
\hline c. $350 \mathrm{G}>\mathrm{A}$ & $\mathrm{R} 117 \mathrm{H}$ & IV & $0.05(21)$ & $0.00(21)$ & $0.07(666)$ & $0.02(19)$ & \\
\hline c. $2657+5 G>A$ & $2789+5 G>A$ & V & $0.25(20)$ & $0.00(20)$ & $0.06(271)$ & & $0.01(21)$ \\
\hline c. $1040 G>A$ & $\mathrm{R} 347 \mathrm{H}$ & & & & $0.06(55)$ & & \\
\hline c. $2988 G>A$ & $3120 G->A$ & & & & $0.06(36)$ & & \\
\hline c. $328 \mathrm{G}>\mathrm{C}$ & D1152H & IV & & & $0.06(124)$ & & \\
\hline c. $3717+12191 C>T$ & $3849+10 \mathrm{kbC}>T$ & V & $0.07(14)$ & $0.00(14)$ & 0.05 (299) & $0.01(42)$ & $0.00(15)$ \\
\hline c. $1364 C>A$ & A455E & V & $0.16(45)$ & $0.01(41)$ & $0.05(109)$ & & \\
\hline c. $1000 C>T$ & R334W & IV & $0.18(11)$ & $0.00(10)$ & $0.05(92)$ & & \\
\hline c.617T>G & L206W & & $0.06(18)$ & $0.05(17)$ & $0.04(52)$ & & \\
\hline c. $3302 T>A$ & M1101K & & & & $0.04(17)$ & & \\
\hline c. $200 C>T$ & P67L & V & $0.07(14)$ & $0.00(14)$ & & & \\
\hline
\end{tabular}

Meconium ileus prevalence (MIP) and pancreas insufficiency prevalence (PIP) scores are presented. Data show the fraction (number) of patients with the genotype. MIP assignments for the Canadian Gene Modifier Consortium (GMC) cohort (MIP Canada) were obtained by rigorous assessment of a subset of patient chart data and were subsequently used to model the probability of meconium ileus for all groups (see Materials and Methods). MIP scores were estimated for individual cystic fibrosis-causing variants occurring with $p$.F508del on the second allele in $n \geq 10$ patients. Discrepant MIP scores between countries may reflect group size differences.

PIP scores were estimated for groups of $n \geq 10$ patients with the same CFTR variants using the Canadian GMC (Canada PIP), as described in ref. 16. As previously reported, CFTR mutations associated with a PIP score $\geq 0.25$ were considered severe mutations, whereas CFTR mutations with a PIP score $<0.25$ were considered mild. The table is sorted based on the largest data set (United States). Because T-tract information was frequently not available, all participants with F508del.R117H were analyzed as a single group. 
from the US ( $r=0.8, P<0.0001)$, German $(r=0.7, P=0.01)$, and North Italian data $(r=0.96, P=0.0008)$ (Supplementary Figure S1 online).

\section{MIP scores distinguish and stratify between "severe" CFTR mutations}

Beyond the categorical separation of severe and mild CF-causing variants, which is paralleled by the PIP score, MIP scores differed among the severe variants. The highest MIP scores were calculated for mutations with premature termination, such as G542X (class I mutation, MIP 0.31), followed by F508del (class II mutation, MIP 0.22); the lowest MIP score was for G551D (class III mutation, MIP 0.08) $(P=$ 0.0009). We were curious to determine whether the low MIP score noted for G551D was obtained for other class III CFTR mutations: those with gating deficiencies. Using the US and Italian data, we calculated equally low MIP scores for G178R (0.09, $n=22)$, S549N $(0.12, n=39), \operatorname{S} 1251 \mathrm{~N}(0.07, n=14)$, and G1244E $(0.0, n=3)$, but not for S549R $(0.21, n=14)$ (Supplementary Table S1 online). The majority of class III CF-causing variants is less severe, per MIP score ranking, when compared with class I or II variants. The US database had the largest number of different CF-causing variants, allowing the estimation of MIP scores for an additional 28 CF-causing variants. The highest MIP score (0.38) corresponded to the CF-causing variant 394delTT (c.262_263delTT) (Table 1). This mutation is found more frequently in the Swedish CF population, where it is associated with lower lung function. ${ }^{23}$ Of interest is that the MIP score ranked the clinically unclassified missense mutation V520F into a similar severe range of CFTR mutations as other class I CF-causing nonsense variants.

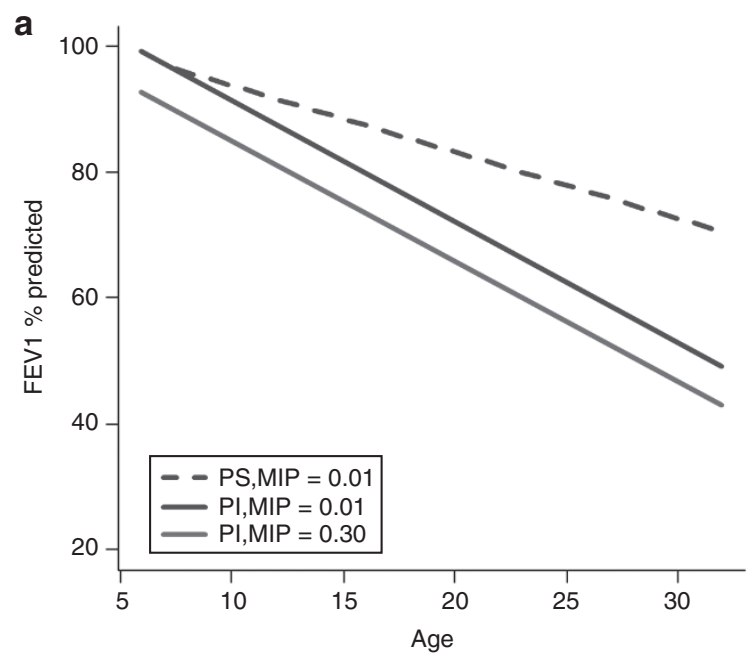

\section{Association of MIP scores and clinical phenotypes}

In a next step we investigated whether the severity of CFTR variants, per MIP scores, corresponds to the severity of disease measured by classical clinical markers such as lung function ( $\mathrm{FEV}_{1} \%$ pred) and nutrition (zBMI).

Lung function (FEV \% pred). There was a significant effect of sex $(P<0.0001)$ and pancreatic status $(P<0.0001)$ on the rate of $\mathrm{FEV}_{1} \%$ pred decline, with an estimated 10 -year rate of decline of $-11.3 \%$ predicted (95\% confidence limits (CLs): $-12.7,-9.9$ ) among patients with PS compared with a 10 -year rate of decline of $-19.9 \%$ predicted (95\% CLs: $-20.3,-19.9$ ) among patients with PI. After adjusting for sex, pancreatic status, and their interaction with age in the model, the MIP score showed an overall association with $\mathrm{FEV}_{1}$ \%pred, with higher MIP scores resulting in lower $\operatorname{FEV}_{1}(P=0.01)$ (Figure 2a). For example, a patient with a MIP score of 0.3 has a $\mathrm{FEV}_{1} \%$ pred value that is $6.6 \%$ (95\% CLs: $\left.1.5 \%, 11.6 \%\right)$ lower than the $\mathrm{FEV}_{1} \%$ pred value of a patient with a MIP score of 0.01 . When the patient's individual MI status was added to the model, it did not show a statistically significant effect $(P=0.7)$, whereas the MIP score remained statistically significant $(P=0.01)$.

Nutrition $(z B M I)$. Similarly, sex $(P<0.0001)$ and pancreatic status $(P<0.0001)$ were also significantly associated with the rate of zBMI decline, with an estimated 10-year rate of decline of -0.13 (95\% CLs: $-0.20,-0.01)$ among patients with PS compared with a 10-year rate of decline of -0.39 (95\% CLs: $-0.45,-0.33$ ) among patients with PI (Figure 2b). After adjusting for sex, the individual pancreatic status, and their interaction with age in the model, the MIP score showed an

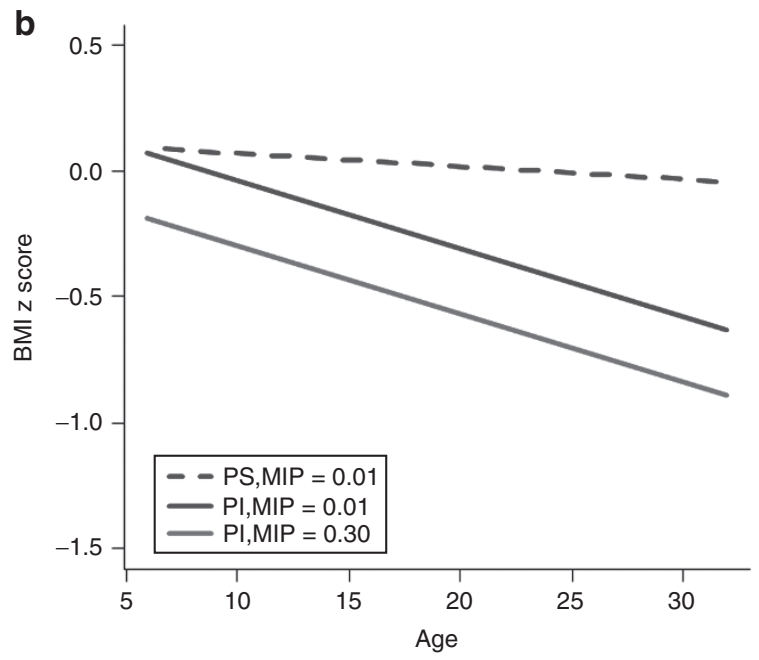

Figure 2 Predicted clinical phenotypes for selected MIP scores. (a) The coefficients obtained from the hierarchical linear regression of percentage of predicted forced expiratory volume in 1 second (FEV1\% pred) across all patients in the Canadian patient data registry (CPDR) were used to predict pulmonary function values for three scenarios representing a range of meconium ileus prevalence (MIP) scores and pancreatic function status. After adjusting for sex and individual pancreatic status in the model, patients with a MIP score of 0.01 had an FEV $\%$ pred $6.6 \%$ higher $(95 \%$ confidence limits (CLs): 1.5, 11.6; $P=0.01)$ than those with a MIP score of 0.3 . (b) The coefficients obtained from the hierarchical linear regression of body mass index z scores (zBMI) across all patients in the CPDR were used to predict nutritional status for three scenarios representing a range of MIP scores and pancreatic function status. After adjusting for sex and the individual pancreatic status in the model, patients with a MIP score of 0.3 had a 0.24 lower zBMI than patients with a MIP score of 0.01 (95\% CLs: $-0.47,-0.01 ; P=0.04)$. 
overall association with zBMI $(P=0.04)$, with a difference in zBMI of 0.23 (95\% CLs: 0.01, 0.47) between a MIP score of 0.01 and 0.3 (Figure $2 \mathbf{b}$ ). When added to the model, the individual MI status demonstrated a significant but clinically minimal association with the rate of $\mathrm{zBMI}$ decline $(P=0.007)$, with a 10-year difference in zBMI change of 0.04 (95\% CLs: 0.01, 0.08 ) between those with and without MI. Including individual $\mathrm{MI}$ and its interaction with age in the model did not affect the association between zBMI and MIP score $(P=0.05)$.

\section{DISCUSSION}

A patient's individual risk for $\mathrm{MI}$ as a first clinical manifestation of CF disease is dependent on the individual CFTR genotype and varies between patients carrying different CF-causing variants. Making use of this genotype-phenotype relationship, we assigned MIP scores to individual CF-causing variants, allowing them to be ranked according to their MI risk. The MIP score also correlated with clinical disease severity measured in other CF-affected organs, suggesting that, on average, variants with high MIP scores also are associated with worse lung disease and BMI. Because MI occurred only in patients carrying "severe" CFTR mutations that are associated with PI, MIP scores seem to be particularly helpful in sorting CF-causing variants within the severe spectrum of CF disease.

The calculated MIP score is well in agreement with previously published information about the functional expression of different CFTR mutations. VanGoor et al. ${ }^{24}$ recently compared the magnitude of mRNA expression levels, the levels of mature CFTR protein, as well as the chloride channel activity of a large number of missense variants using a Fisher rat thyroid cell expression system. Comparing findings with ours, we observe that generally, the MIP score correlates with the CFTR functional levels $(P=0.009$; Table 2). The mutations M1101K and R347P, however, showed no CFTR function, with a low MIP score and intermediate PIP score, suggesting that the functional consequences of these mutations may be very organ-specific and/or are greatly influenced by non-CFTR-modifying factors. This finding does seem to reflect previous reports of various outcomes of patients with M1101K or R347P, ranging from PI and an early decline in lung function to PS and only mild lung disease. . $^{25,26}$

MIP scores distinguished between the "molecular" classification of CFTR mutations, especially regarding the distinctive class III or gating mutations. The highest MIP scores were calculated for class I mutations, including nonsense variants, the missense variant V520F, and the Scandinavian variant 394delTT. Nonsense variants and c.262_263delTT have been associated with a more severe CF phenotype when compared with F508del. ${ }^{23,27}$ Little clinical information is available for V520F, but the location of the amino acid substitution may indicate impairment of channel function.

MIP scores were significantly different between the mutations F508del and G551D, which is in agreement with early studies of smaller numbers of patients with CF that reported a lower MI incidence in patients carrying F508del/G551D when compared with patients with CF with F508del/ F508del. ${ }^{28,29}$ We demonstrated equally low MIP scores for other class III CFTR mutations (G178R, S549N, G1244E, S1251N), further supporting the idea that class III CFTR mutations are not as severe as F508del, at least with respect to gastrointestinal development. This difference in the clinical phenotype is somewhat remarkable given that G551D is classically viewed as a "nongating" and "nonfunctional" CFTR mutation. Defects in responsiveness to adenosine triphosphate regulation is thought to explain the low open probability of G551D channels..$^{30,31}$ However, following expression in different heterologous cells, measurable G551D-mediated chloride currents have been reported. ${ }^{32}$ In Fisher rat thyroid cells G551D showed 1\% of wild-type function, and human bronchial epithelial cells generated from G551D/F508del lung explants expressed 5\% wild-type CFTR function. ${ }^{24}$ Our results suggest that, in contrast to classes I and II, class III CFTR mutations confer sufficient chloride channel function to maintain early intestinal fluid homeostasis and thus eliminate the risk for $\mathrm{MI}$ in patients with $\mathrm{CF}$.

The MIP score is indicative of the clinical severity of specific CFTR mutations; it is not intended to predict outcome on an individual patient level, but it can be used to evaluate larger patient groups. While non-CFTR modifier genes as well as environmental factors largely influence the development and progression of lung disease and nutritional decline, ${ }^{33-36}$ we demonstrate that the severity of the underlying CFTR genotype

Table 2 Meconium ileus prevalence scores and CFTR function

\begin{tabular}{lcc} 
CFTR mutation & MIP score & CFTR function (\%wt) \\
\hline High MIP score & & 0.2 \\
V520F & 0.38 & 0.5 \\
N1303K & 0.25 & 0.4 \\
F508del & 0.27 & 0.1 \\
R560T & 0.27 & 0 \\
A559T & 0.24 & 1 \\
G551D & 0.15 & 0.8 \\
G85E & 0.14 & 0 \\
R1066C & 0.13 & \\
Low MIP score & & 0 \\
R347P & 0.1 & 2.9 \\
R117C & 0.1 & 33 \\
R117H & 0.07 & 5 \\
R347H & 0.06 & 1.3 \\
R334W & 0.05 & 6 \\
A455E & 0.05 & 5 \\
L206W & 0.04 & 0 \\
M1101K & 0.04 & 8 \\
P67L & 0.0 & 0 \\
\hline
\end{tabular}

The table compares meconium ileus prevalence (MIP) scores and measured cystic fibrosis transmembrane conductance regulator (CFTR) function in Fisher rat thyroid determined by VanGoor et al. ${ }^{24}$ for the major and missense cystic fibrosis-causing variants for which patient group size was $\geq 10$ in at least the US group. Following exclusion of R117H (the Fisher rat thyroid studies would not have accommodated haplotype considerations), MIP scores correlated with CFTR function ( $r=-0.63$; $95 \% \mathrm{Cl}-0.86$ to $-0.19 ; P=0.009)$. 


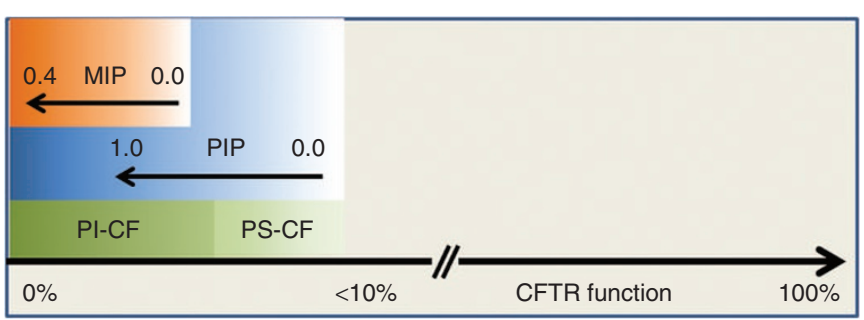

Figure 3 Schematic ranking of CFTR mutation using meconium ileus prevalence (MIP) and pancreas insufficiency prevalence (PIP) scores. PIP scores have been published previously. ${ }^{18}$

itself plays a role in predisposing patients to an overall better or worse clinical outcome measured as lung function and nutritional status.

The high agreement of MIP scores among different CF patient registries indicates that the risk for MI can be used as a phenotypic marker for the severity of CFTR mutations. This reflects the heritability of susceptibility ${ }^{8}$ and a presumption that early occurrence equates to minimal environmental interference. However, that prenatal toxic drug exposure can lead to measurable drug levels in the meconium of infants $\mathrm{s}^{37,38}$ may indicate that some environmental contribution does occur. A CF pig model further supports the MIP score assignments as a consistent linear relation between intestinal CFTR expression and MI susceptibility ${ }^{39}$ (with a containment of genetic background). Population studies with large patient cohorts have identified that non-CFTR genes such as SLC6A14, SLC9A3, and SLC26A9 are associated with, and do modify, MI susceptibility. Other studies, including family-based investigations, have also highlighted that loci on chromosomes 19 (ref. 40) and 8 (specifically the MSRA gene $)^{41}$ influence MI susceptibility. Collectively, it is apparent that the individual risk for $\mathrm{MI}$ in a patient with $\mathrm{CF}$ depends on the severity of the CFTR gene defect plus the influence of modifier genes. A parallel scenario has been proposed for the intestinal obstruction observed in the CF mouse and ferret genetic models. ${ }^{40,42}$

For most CF-causing variants, information about the phenotype or the severity of the disease course is unknown. This has prompted the establishment of CFTR2 as a public resource with summarized clinical data, including $\mathrm{FEV}_{1} \%$ pred, frequencies of Pseudomonas aeruginosa infection, sweat chloride results as well as information about the pancreas status. ${ }^{11}$ While knowledge of these descriptions provides averages of expected clinical phenotypes for a specific CFTR variant, it does not allow discrimination of disease severity by organ. In addition, the prediction of the clinical consequences of specific CFTR variants based on nucleotide sequence or in vitro assays remains immensely challenging. In silico tools are often insufficient to accurately predict the liability of many CFTR variants, ${ }^{18}$ leaving the interpretation of the likelihood of specific clinical consequences to highly experienced CF specialists. Notably, as shown in the schematic in (Figure 3), MIP and PIP scores offer additional tools to estimate the clinical severity of CFTR variants based on phenotype presentation.
Thus, MIP and PIP scores can be used to benchmark already existing in silico tools and molecular measures, and help to explain complex genotype-phenotype correlations. Of immediate interest is understanding how gating mutations limit susceptibility to MI.

A major strength of this study was the use of large cohorts, enabling determination of phenotypic scores in rare conditions with a range of disease-causing variants, such as CF. This study is strengthened by the use of the very rigorously controlled patient sets (GMC and Canadian patient data registry) to develop a model to estimate the probability of MI and to develop the MIP score. We were not able to apply the same rigor to control the meconium and pancreas phenotypes for the other CF patient data registries; rather, we applied the estimated MI probability model. This may have led to subtle over- or underestimations of MI for some CFTR variant groups in the other databases. Nevertheless, the strong correlation between the MIP scores from the GMC and the other databases argues against a large error in our MIP calculations. A noted limitation remains the absence of complete genotypes for a large portion of registered patients with CF (noted in all databases), which may have led to some incongruence between derived MIP scores.

In summary, we developed the MIP score as a phenotypebased scoring system to help sort CFTR variants according to their clinical disease severity. MIP and PIP scores advance our knowledge regarding the genotype-phenotype relation in CF and help to stratify patients according to their presumed disease severity for future clinical or genetic studies.

\section{SUPPLEMENTARY MATERIAL}

Supplementary material is linked to the online version of the paper at http://www.nature.com/gim

\section{ACKNOWLEDGMENTS}

This study was funded by Cystic Fibrosis Canada. The authors thank the steering committees of the Canadian patient data registry, the US patient registry, the German CF patient registry, and the CF patient data set from Veneto/Trentino/Alto Adige for sharing their data sets.

\section{DISCLOSURE}

The authors declare no conflict of interest.

\section{REFERENCES}

1. Carlyle BE, Borowitz DS, Glick PL. A review of pathophysiology and management of fetuses and neonates with meconium ileus for the pediatric surgeon. J Pediatr Surg 2012;47:772-781.

2. Kerem E, Corey M, Kerem B, Durie P, Tsui LC, Levison H. Clinical and genetic comparisons of patients with cystic fibrosis, with or without meconium ileus. J Pediatr 1989;114:767-773.

3. Efrati $\mathrm{O}$, Nir J, Fraser $\mathrm{D}$, et al. Meconium ileus in patients with cystic fibrosis is not a risk factor for clinical deterioration and survival: the Israeli Multicenter Study. J Pediatr Gastroenterol Nutr 2010;50:173-178.

4. Kerem E, Corey M, Kerem BS, et al. The relation between genotype and phenotype in cystic fibrosis-analysis of the most common mutation (delta F508). N Engl J Med 1990;323:1517-1522.

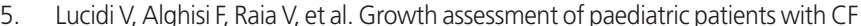
comparing different auxologic indicators: A multicentre Italian study. J Pediatr Gastroenterol Nutr 2009;49:335-342. 
6. Lai HJ, Cheng Y, Cho H, Kosorok MR, Farrell PM. Association between initial disease presentation, lung disease outcomes, and survival in patients with cystic fibrosis. Am J Epidemiol 2004;159:537-546

7. Mornet E, Simon-Bouy B, Serre JL, et al. Genetic differences between cystic fibrosis with and without meconium ileus. Lancet 1988;1:376-378.

8. Blackman SM, Deering-Brose R, McWilliams R, et al. Relative contribution of genetic and nongenetic modifiers to intestinal obstruction in cystic fibrosis. Gastroenterology 2006;131:1030-1039.

9. Sun L, Rommens JM, Corvol H, et al. Multiple apical plasma membrane constituents are associated with susceptibility to meconium ileus in individuals with cystic fibrosis. Nat Genet 2012;44:562-569.

10. Correlation between genotype and phenotype in patients with cystic fibrosis. The Cystic Fibrosis Genotype-Phenotype Consortium. N Engl J Med 1993;329:1308-1313.

11. Sosnay PR, Siklosi KR, Van Goor F, et al. Defining the disease liability of variants in the cystic fibrosis transmembrane conductance regulator gene. Nat Genet 2013;45:1160-1167.

12. Ahmed N, Corey M, Forstner G, et al. Molecular consequences of cystic fibrosis transmembrane regulator (CFTR) gene mutations in the exocrine pancreas. Gut 2003;52:1159-1164.

13. Kristidis P, Bozon D, Corey M, et al. Genetic determination of exocrine pancreatic function in cystic fibrosis. Am J Hum Genet 1992;50:1178-1184.

14. Welsh MJ, Smith AE. Molecular mechanisms of CFTR chloride channel dysfunction in cystic fibrosis. Cell 1993;73:1251-1254.

15. Hirtz S, Gonska T, Seydewitz HH, et al. CFTR Cl- channel function in native human colon correlates with the genotype and phenotype in cystic fibrosis. Gastroenterology 2004;127:1085-1095.

16. Ooi CY, Dorfman R, Cipolli M, et al. Type of CFTR mutation determines risk of pancreatitis in patients with cystic fibrosis. Gastroenterology 2011;140:153-161.

17. Terlizzi $\mathrm{V}$, Tosco A, Tomaiuolo $R$, et al. Prediction of acute pancreatitis risk based on PIP score in children with cystic fibrosis. J Cyst Fibros 2014;13:579-584.

18. Dorfman R, Nalpathamkalam T, Taylor C, et al. Do common in silico tools predict the clinical consequences of amino-acid substitutions in the CFTR gene? Clin Genet 2010;77:464-473.

19. Li W, Sun L, Corey M, et al. Understanding the population structure of North American patients with cystic fibrosis. Clin Genet 2011;79:136-146.

20. Hankinson JL, Odencrantz JR, Fedan KB. Spirometric reference values from a sample of the general U.S. population. Am J Respir Crit Care Med 1999;159:179-187.

21. Wang $X$, Dockery DW, Wypij D, Fay ME, Ferris BG Jr. Pulmonary function between 6 and 18 years of age. Pediatr Pulmonol 1993;15:75-88.

22. Bryk ASR. Application of hierachical linear models to assessing change. Psychol Bull 1987;101:147-158.

23. Geborek A, Hjelte L. Association between genotype and pulmonary phenotype in cystic fibrosis patients with severe mutations. J Cyst Fibros 2011;10:187-192.

24. Van Goor F, Yu H, Burton B, Hoffman BJ. Effect of ivacaftor on CFTR forms with missense mutations associated with defects in protein processing or function. J Cyst Fibros 2014;13:29-36.

25. Varon R, Stuhrmann M, Macek M Jr, et al. Pancreatic insufficiency and pulmonary disease in German and Slavic cystic fibrosis patients with the R347P mutation. Hum Mutat 1995;6:219-225.

26. Zielenski J, Fujiwara TM, Markiewicz D, et al. Identification of the M1101K mutation in the cystic fibrosis transmembrane conductance regulator (CFTR) gene and complete detection of cystic fibrosis mutations in the Hutterite population. Am J Hum Genet 1993;52:609-615.

27. Shoshani T, Augarten A, Gazit E, et al. Association of a nonsense mutation (W1282X), the most common mutation in the Ashkenazi Jewish cystic fibrosis patients in Israel, with presentation of severe disease. Am J Hum Genet 1992;50:222-228.

28. Hamosh A, King TM, Rosenstein BJ, et al. Cystic fibrosis patients bearing both the common missense mutation Gly_Asp at codon 551 and the delta F508 mutation are clinically indistinguishable from delta F508 homozygotes, except for decreased risk of meconium ileus. Am J Hum Genet 1992;51:245-250.

29. Feingold J, Guilloud-Bataille M. Genetic comparisons of patients with cystic fibrosis with or without meconium ileus. Clinical Centers of the French CF Registry. Ann Genet 1999;42:147-150.

30. Bompadre SG, Sohma Y, Li M, Hwang TC. G551D and G1349D, two CF-associated mutations in the signature sequences of CFTR, exhibit distinct gating defects. J Gen Physiol 2007;129:285-298.

31. Pasyk S, Li C, Ramjeesingh M, Bear CE. Direct interaction of a small-molecule modulator with G551D-CFTR, a cystic fibrosis-causing mutation associated with severe disease. Biochem J 2009;418:185-190.

32. Fulmer SB, Schwiebert EM, Morales MM, Guggino WB, Cutting GR. Two cystic fibrosis transmembrane conductance regulator mutations have different effects on both pulmonary phenotype and regulation of outwardly rectified chloride currents. Proc Natl Acad Sci USA 1995;92:6832-6836.

33. Li W, Soave D, Miller MR, et al. Unraveling the complex genetic model for cystic fibrosis: pleiotropic effects of modifier genes on early cystic fibrosis-related morbidities. Hum Genet 2014;133:151-161.

34. Bradley GM, Blackman SM, Watson CP, Doshi VK, Cutting GR. Genetic modifiers of nutritional status in cystic fibrosis. Am J Clin Nutr 2012;96:1299-1308.

35. McColley SA, Ren CL, Schechter MS, Regelmann WE, Pasta DJ, Konstan MW; Epidemiologic Study of Cystic Fibrosis. Risk factors for onset of persistent respiratory symptoms in children with cystic fibrosis. Pediatr Pulmonol 2012:47:966-972.

36. Vandenbranden SL, McMullen A, Schechter MS, et al.; Investigators and Coordinators of the Epidemiologic Study of Cystic Fibrosis. Lung function decline from adolescence to young adulthood in cystic fibrosis. Pediatr Pulmonol 2012;47:135-143.

37. Arria AM, Derauf C, Lagasse $L L$, et al. Methamphetamine and other substance use during pregnancy: preliminary estimates from the Infant Development, Environment, and Lifestyle (IDEAL) study. Matern Child Health J 2006;10: 293-302.

38. Gray TR, Kelly T, LaGasse LL, et al. New meconium biomarkers of prenatal methamphetamine exposure increase identification of affected neonates. Clin Chem 2010;56:856-860.

39. Stoltz DA, Rokhlina T, Ernst SE, et al. Intestinal CFTR expression alleviates meconium ileus in cystic fibrosis pigs. J Clin Invest 2013;123:2685-2693.

40. Zielenski J, Corey M, Rozmahel R, et al. Detection of a cystic fibrosis modifier locus for meconium ileus on human chromosome 19q13. Nat Genet 1999;22:128-129.

41. Henderson LB, Doshi VK, Blackman SM, et al. Variation in MSRA modifies risk of neonatal intestinal obstruction in cystic fibrosis. PLOS Genet 2012;8:e1002580

42. Sun X, Sui H, Fisher JT, et al. Disease phenotype of a ferret CFTR-knockout model of cystic fibrosis. J Clin Invest 2010;120:3149-3160. 